Int. J. Odontostomat., 9(1):79-84, 2015.

\title{
Microbiota Oral Presente en Pacientes Edentulos
}

\author{
Oral Microbiota in Edentulous Patients
}

Karla Gabriela Ocampo García* \& Julio Basilio Robles*

OCAMPO, G. K. G. \& BASILIO, R. J. Microbiota oral presente en pacientes edentulos. Int. J. Odontostomat., 9(1):79-84, 2015.

RESUMEN: El objetivo fue identificar las condiciones orales de pacientes edentulos que acudieron a rehabilitación a la Clínica de Prótesis Total de la Facultad de Odontología de la Universidad Autónoma del Estado de México. El diseño del estudio fue observacional, descriptivo, transversal y prospectivo con una muestra no probabilística de oportunidad y secuencial para estudiar en 160 pacientes el tipo de flora microbiana, portadores de prótesis orales. Los resultados reportan que el promedio de edad para la mayor población edéntula está entre los 56 y 65 años de edad. En relación a las patologías existentes en boca se encontró que el $80 \%$ de la población no presentaba signos o síntomas, del $20 \%$ eran sintomáticos, todos asociados a estomatitis subprotésica y en 2 de estos pacientes se diagnosticó síndrome de boca ardiente. En relación a la flora microbiana de la cavidad oral encontramos diversidad de microorganismos como: $17 \%$ para la flora cocoide Gram+, $2 \%$ para flora bacilar Gram-, $17 \%$ de presencia de leucocitos, $18 \%$ de bacterias, $18 \%$ de células epiteliales, $2 \%$ de diplococos, $5 \%$ de Staphylococcus, $7 \%$ de tétradas, $7 \%$ de micrococos, $5 \%$ de Streptococcus, $2 \%$ de enterobactereceas. No se encontraron Candida albicans ni levaduras. El sexo femenino el que presenta mayor tendencia a padecer edentulismo, a razón demográfica nacional que señala que hay más mujeres que hombres. En el apartado de microflora oral se identificó una gran cantidad de microorganismos abundando la flora bacteriana Gram+, pero se identifican varios organismos anaerobios que convierten la cavidad oral en un medio de cultivo, lo cual deberá correlacionarse con la edad y presencia de enfermedades sistémicas concomitantes, ya que microorganismos como el Staphylococcus y Streptococcus pueden tener una repercusión a nivel sistémico, tal y como se demuestra en la asociación de edentulismo a enfermedades cardiovasculares y metabólicas.

PALABRAS CLAVE: edentulismo, condiciones orales, flora microbiana oral.

\section{INTRODUCCIÓN}

Partiendo de la definición del término condición oral, como el estado oral que se encuentra presente bajo ciertas características y propiedades físico, químico y biológicas que a su vez puede ser variable o constante, llevamos a cabo una investigación a través de la cual se pudieran establecer las condiciones orales de los pacientes edéntulos que acuden a la clínica de prótesis total de la Facultad de Odontología de la Universidad Autónoma del Estado de México con la finalidad de ofrecer tanto mejores servicios de diagnóstico como de pronostico así mismo un plan de tratamiento acertado, teniendo como interés la salud general del paciente y al mismo tiempo la prevención de otras alteraciones orales (Bagg, 1999; Burnett et al., 1986; Danser et al., 1997).

Las principales consecuencias del edentulismo parcial son la reabsorción del hueso alveolar o el des- plazamiento de los de los dientes vecinos hacia el nuevo espacio creado. El movimiento de los dientes genera alteraciones en la oclusión (Bagg; Burnett et al.). Esta condición física también tiende a generar una disminución en el autoestima, inducir alteraciones en la pronunciación de las palabras, en la función masticatoria y en la articulación temporomandibular (Devides \& Franco, 2006; Dinatale \& Guilarte, 2009; Esponda Vila, 1993; Koeck, 2007; Kraus et al., 1981).

El edentulismo es generalmente asociado al incremento en la edad, principalmente asociado a pacientes geriatras, por lo que parte de las condiciones orales del paciente edéntulo corresponden a pacientes adultos mayores; es el caso de la actividad muscular que presenta una disminución en la fuerza por la reducción significativa de la densidad de los músculos masetero y pterigoideo (McCord \& Grant, 2000).

*Facultad de Odontología, Universidad Autónoma del Estado de México, Toluca, México. 
La secreción de saliva, en lo que respecta a la secreción submandibular y sublingual, disminuye con la edad hasta un $25 \%$, que corresponde a la incidencia de la xerostomía (Dinatale \& Guilarte; Esponda Vila; Nolte, 1982; OPS, 2004). En los pacientes desdentados, que no poseen receptores periodontales, se observa un reflejo masticación-parótida. En este caso, las terminaciones nerviosas aferentes en la mucosa debajo de las prótesis orales podrían tomar esta función para mantener el reflejo (OPS).

En cuanto a los huesos maxilar y mandibular, en promedio después de 20 a 23 años sin dientes, los procesos alveolares se encuentran reabsorbidos casi por completo y sustituidos por tejido conectivo denso llamada eminencia flácida. Por lo que la función de soporte recae sobre el hueso palatino, cresta cigomáticoalveolar y espina nasal en el caso del maxilar. Para la mandíbula, dicha función recae sobre la línea oblicua de la rama de la mandíbula, cresta milohioidea, protuberancia y espina mentoniana (Kraus et al.).

La flora de la cavidad oral es un reflejo del medio; su naturaleza depende de los requerimientos fisicoquímicos y nutricionales, y son proporcionales a la dieta del huésped, los tejidos y la presencia de otros microorganismos (Kraus et al.; McCord \& Grant). En el paciente edéntulo se crea un desequilibrio microbiológico debido a la edad avanzada, incubación de la mucosa oral bajo la base de la prótesis y en ocasiones la insuficiente higiene oral. En los portadores de prótesis dominan los lactobacilos, Streptococcus y Candida albicans (Botero et al., 2005; Ozawa Deguchi, 1995; Regezi \& Sciubba, 2004.

Entre las patologías más comúnmente descritas para pacientes edéntulos se encuentra la leucoplasia, el épulis fisurado, mucositis, xerostomía y estomatitis protésica para los portadores ya de prótesis totales.

\section{MATERIAL Y MÉTODO}

Se realizó una estudio observacional, descriptivo, transversal, con una muestra no probabilística de oportunidad y secuencial para identificar las condiciones orales en 160 pacientes edéntulos que acudieron a rehabilitación a la Clínica de Prótesis Total de la Facultad de Odontología de la Universidad Autónoma del Estado de México. Se valoró la presencia de estomatitis subprotésica o alguna patología oral anexa, así como sus signos y síntomas; el tipo de microbiota habitual del paciente edéntulo a través de pruebas para la identificación de los microorganismos aislados y purificados, iniciando con el examen microscópico previa coloración y la observación macroscópica de las colonias, seguido del análisis a través de pruebas bioquímicas convencionales o por pruebas rápidas que incluyen sistemas microbioquímicos y enzimáticos manuales o automatizados, que permiten la identificación definitiva de la microbiota oral.

Se recabó la información mediante un formato en función a las características de nuestro objeto de estudio, basado en conocer las condiciones orales del paciente edéntulo, con la utilidad del método descriptivo para establecer criterios diferenciales entre los pacientes y conocer qué condiciones orales son las más frecuentes entre los pacientes edéntulos (Figs. 1 y 2 ).

Utilizando las variables de la investigación se realizó una estadística inferencial a través del programa SPSS, donde se relacionó edad, sexo, portador de prótesis y tipo de microflora oralal entre sí.

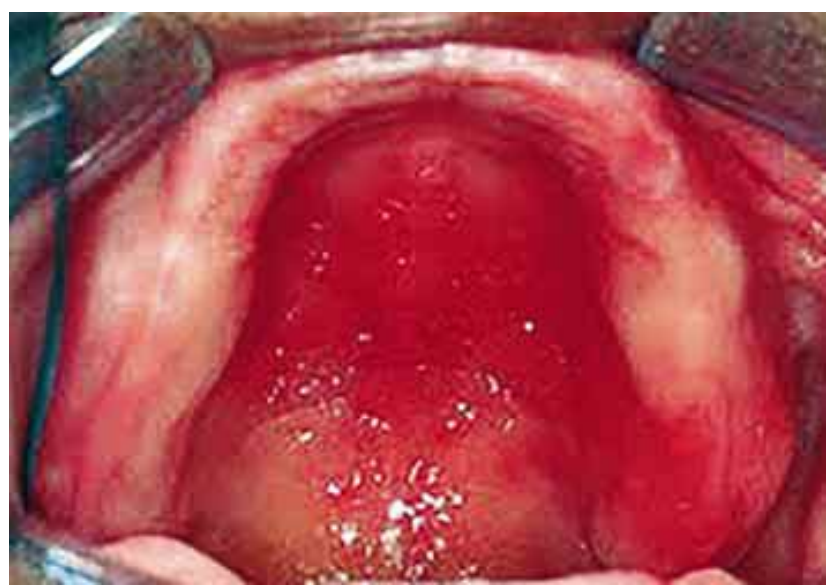

Fig. 1. Muestra reborde alveolar del paciente edéntulo.

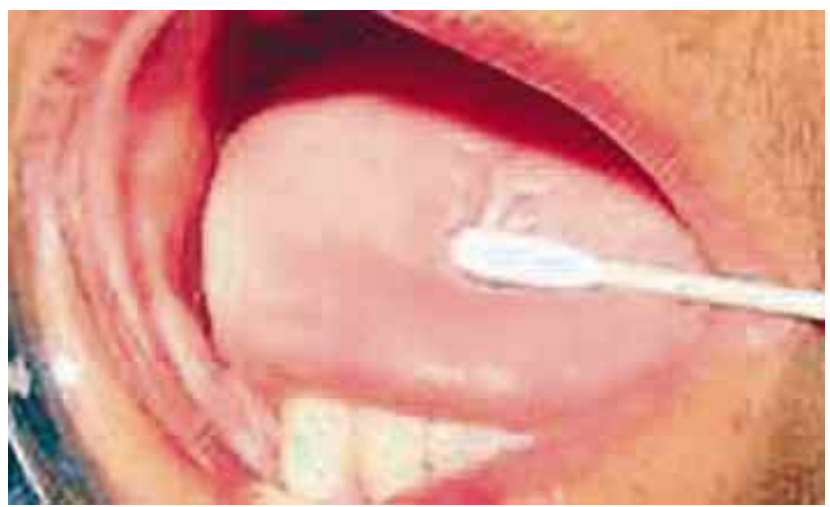

Fig. 2. Toma de muestra para evaluar la microflora en cavidad oral de pacientes edéntulos. 


\section{RESULTADOS}

Los resultados reportan que el promedio de edad (Fig. 3) para la mayor población edéntula está entre los 56 y 65 años de edad con un $81 \%$ (131 pacientes), en cuestión de género se determinó que el $52 \%$ (83 pacientes) de los pacientes edéntulos corresponde al sexo femenino, solo el 58\% (94 pacientes) de la población utiliza prótesis total removible para sustituir la falta de dientes y de estos el 48\% (45 pacientes) ha utilizado sus prótesis por más de 16 años, y de estos 94 pacientes portadores de prótesis dentales solo el $54 \%$ (50 pacientes) tiene una adecuada técnica de uso e higiene.

En relación a las patologías existentes en la cavidad oral, se encontró que el 80\% (128 pacientes) de la población no presentaba signos o sínto-

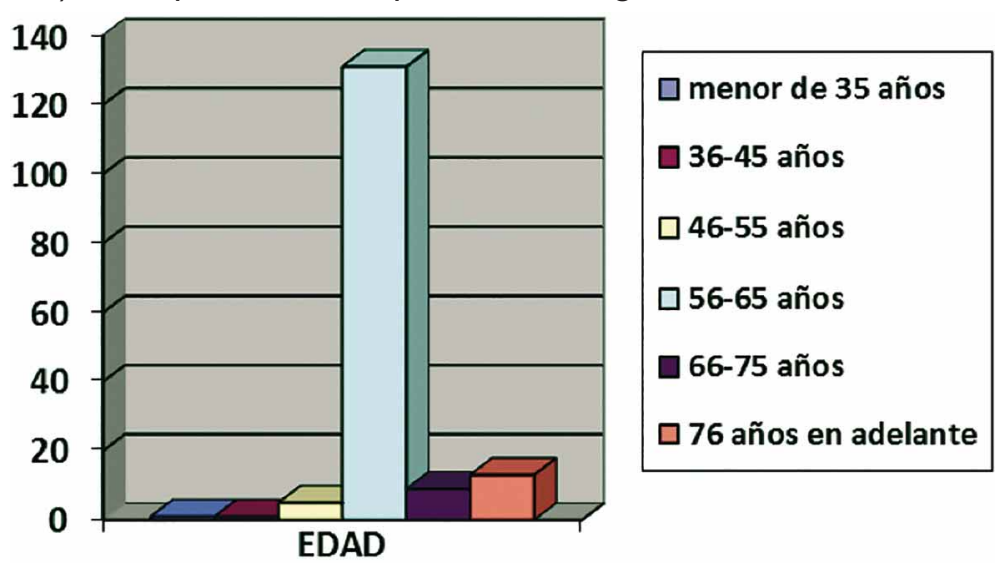

Fig. 3. Promedio de edad para la población edéntula analizada.

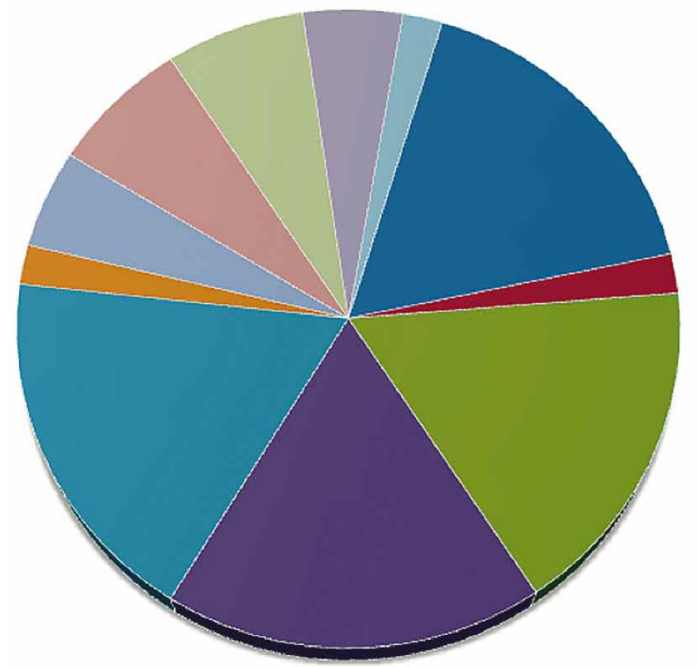

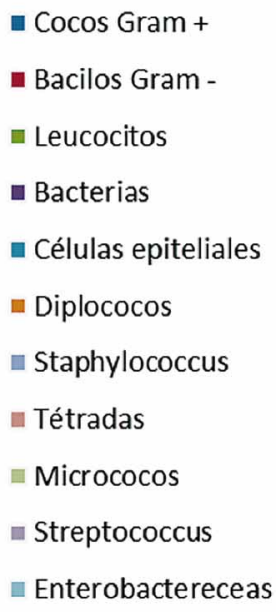

Fig. 4. Microorganismos encontrados en la microbiota oral. mas, del $20 \%$ restante (32 pacientes) los síntomas que más reconocen es el dolor con un 50\% (16 pacientes), las úlceras en un 30\% (9 pacientes) y el sangrado y la inflamación en un 10\% (3 pacientes) respectivamente, asociados a estomatitis subprotésica en 30 pacientes, $93 \%$, como la patología oral más frecuentemente reportada y en 2 de estos 32 pacientes $(7 \%)$ se diagnosticó síndrome de boca ardiente (urente). En relación a la flora microbiana de la cavidad oral encontramos una gran variedad de microorganismos los cuales se desglosa de la siguiente manera: $17 \%$ para la flora cocoide Gram,$+ 2 \%$ para flora bacilar Gram-, $17 \%$ de presencia de leucocitos, $18 \%$ de bacterias, $18 \%$ de células epiteliales, $2 \%$ de diplococos, $5 \%$ de Staphylococcus, $7 \%$ de tétradas, $7 \%$ de micrococos, $5 \%$ de Streptococcus, $2 \%$ de enterobactereceas (Fig. 4). No se encontraron Candida albicans ni levaduras.

Correlación Sexo-Tipo de Microflora. No existe correlación significativa en ningún caso, exceptuando uno, el de sexo - Leucocitos. En este caso el coeficiente de correlación fue de $-0,261$, siendo significativo a un nivel de 0,05 , por lo que es un análisis confiable. Podemos decir que no existe una correlación entre el sexo y la presencia de leucocitos, lo más que podemos decir es que existe una tendencia muy baja (casi imperceptible) del sexo masculino a tener más leucocitos que el sexo femenino, aunque esta tendencia no llega a ser válida estadísticamente, ya que no alcanzó el nivel de 0,8 .

\section{Correlación Edad - Tipo de Microflora.} No existe correlación significativa en ningún caso, exceptuando uno, el de edad - bacterias. En este caso correlacionamos la edad con tres valores de presencia de bacterias: escasa, regular y abundante. El coeficiente de correlación fue de 0,378 , siendo significativo a un nivel de 0,01 , por lo que es un análisis confiable. Podemos decir que no existe una correlación entre la edad y la presencia de bacterias, sin embargo podemos decir que, aparentemente, a mayor edad mayor presencia de bacterias, aunque esta tendencia no llega a ser válida estadísticamente, ya que no alcanza el nivel de 0,8 . 
Correlación Portador de Prótesis - Edad. El coeficiente de correlación fue de $-0,432$, siendo significativo a un nivel de 0,01 , por lo que es un análisis confiable. Podemos decir que no existe una correlación entre los portadores de prótesis y la edad. Lo más que podemos decir es que existe una tendencia de que a mayor edad, menor cantidad de portadores de prótesis.

Haciendo una lectura adecuada de los datos, nos damos cuenta que esta aseveración no es válida en ningún sentido, ya que la distribución de los mismos no cumple con los estándares mínimos para un análisis de estas características; la disparidad de nuestra muestra no lo permite. Existen muy pocos sujetos en los niveles más bajos de edad, y estos van en aumento al elevarse la misma, es por ello que estos resultados son engañosos a primera vista.

Correlación Portador de Prótesis - Sexo. El coeficiente de correlación fue de $-0,290$. Esta correlación es significativa a nivel de 0,05 , por lo que es confiable. Conforme los datos, podemos decir que no existe una correlación entre el sexo y la portación de prótesis. Podemos decir que existe una tendencia del sexo femenino a portar prótesis más que el sexo masculino, aunque esta tendencia no llega a ser válida estadísticamente, ya que no alcanza el nivel de 0,8 .

\section{DISCUSIÓN}

En esta investigación pudimos establecer que partiendo de los datos obtenidos de la investigación y conforme a las características de la muestra que no hay presencia de alteraciones patológicas orales relacionadas directamente con el edentulismo, ya que de 160 personas que componen nuestro universo de estudio, 128 pacientes no presentan ninguna patología, lo coincidente con la postura de Kostecka (1924) al observar en sus estudios que la flora anaeróbica dependía de la erupción dentaria y disminuía con la perdida de ellos y produce una disminución en el número de bacterias orales, pero el uso de dentaduras artificiales cambia el medio oral transformándolo en un ambiente anaerobio (Kraus et al.; McCord \& Grant).

La pertinencia de estos datos nos permite superar la clásica dicotomía de que la ausencia de una adecuada higiene oral es causa directa de la perdida de los dientes, ésta discusión es rebasada por su obviedad; lo realmente interesante es coincidir en que hay una marcada tendencia de pacientes edéntulos con enfermedades sistémicas. Así lo señala la investigación de Andrade Lima, al reconocer que cada vez son más las investigaciones que descubren que las infecciones buco-dentales se asocian con enfermedades sistémicas, entre ellas alteraciones cerebrovasculares, respiratorias, diabetes mellitus, hipertensión arterial y las bacterias Gram- viables del biofilm y citoquinas pro-inflamatorias que pueden ingresar al torrente sanguíneo e influir en la salud general y susceptibilidad a ciertas enfermedades implicando factores de riesgo. También encontramos en coincidencia que las condiciones orales de los pacientes edéntulos son factor clave para el uso y tratamiento de prótesis totales. Por lo tanto la calidad de vida de una persona que presenta edentulismo es directamente proporcional al deterioro general de su salud, agravada a la falta de cuidado e higiene oral, pues con nuestra investigación comprobamos que de los 160 pacientes revisados, solo el $31 \%$ de los pacientes presentaba una adecuada higiene oral; lo cual redunda en una disminución de su calidad de vida.

En relación a la flora microbiana de la cavidad oral encontramos una gran variedad de microorganismos siendo los hallados los siguientes: $17 \%$ para la flora cocoide Gram,$+ 2 \%$ para flora bacilar Gram-, $17 \%$ de presencia de leucocitos, $18 \%$ de bacterias, $18 \%$ de células epiteliales, $2 \%$ de diplococos, $5 \%$ de Staphylococcus, $7 \%$ de tétradas, $7 \%$ de micrococos, $5 \%$ de Streptococcus, $2 \%$ de enterobactereceas. No se encontraron Candida albicans ni levaduras. Sin embargo los resultados del análisis del SPSS nos señala que no existe correlación al establecer el cruce de variables edad-sexo, exceptuando uno, el de sexo - Leucocitos. En este caso el coeficiente de correlación es de $-0,261$, siendo significativo a un nivel de 0,05 , por lo que no es un análisis confiable. Podemos decir que existe una tendencia muy baja (casi imperceptible) del sexo masculino a tener más leucocitos que el sexo femenino, aunque esta tendencia no llega a ser válida estadísticamente, ya que no alcanza el nivel de 0.8 , esto mismo se puede deber al factor del edentulismo que señala que con la perdida de los órganos dentarios se produce una disminución en el número de bacterias orales.

\section{CONCLUSIONES}

Con el envejecimiento se presentan múltiples cambios fisiológicos como la disminución en la fuerza 
de la mordida por la falta de tonicidad en músculos masticadores derivado de una reducción significativa de la densidad de los músculos masetero y pterigoideo y una disminución generalizada de las fibras c reactivas. En los pacientes desdentados, que no poseen receptores periodontales, se observa un reflejo masticación-parótida. En este caso, las terminaciones nerviosas aferentes en la mucosa debajo de las prótesis orales podrían tomar esta función para mantener el reflejo, dado que las prótesis completamente mucosoportadas, la importancia rehabilitadora de la prótesis total esta en recuperar las funciones de la boca que son: primero una masticación eficaz sin que la prótesis interfiera en la deglución, puesto que ambas funciones influyen directamente en algo tan fundamental como lo es la alimentación, y segundo una fonética adecuada que permita al paciente una correcta comunicación, sin que la prótesis interfiera. Por lo cual, es importante la rehabilitación protésica intraoral en pacientes edéntulos.

En nuestro estudio se identificó una gran cantidad de microorganismos abundando la flora bacteriana Gram+, asi como varios organismos anaerobios que convierten la cavidad oral en un medio de cultivo, por lo que al tomar en cuenta que los pacientes son adultos mayores con predisposición a enfermedades sistémicas, es imperativo equilibrar y atender la situa- ción resultante de la microbiota del paciente edéntulo, tanto en tratamiento como en prevención dados los reportes positivos de Staphylococcus y Streptococcus que puedan tener una repercusión a nivel sistémico.

Los pacientes edéntulos que analizamos en la clínica de prótesis total de la facultad de Odontología de la UAEM no presentan patologías orales en su mayoría de gran impacto, solo las relacionadas al uso de prótesis totales como son la estomatitis subprotésica debido a la falta de higiene, lo que sugiere llevar a cabo un estudio prospectivo que correlacione la microbiota de la prótesis en la influencia de aparición de esta y otras patologías orales.

Así mismo la muestra reitera que es el sexo femenino el que presenta mayor tendencia a padecer edentulismo con un $52 \%$ por encima del sexo masculino, a razón demográfica nacional que señala que hay más mujeres que hombres.

Dados los hallazgos de nuestro estudio podríamos concluir que para que exista un equilibrio entre el huésped edéntulo y la microbiota oral se conjuntan varios factores, entre los que destacan la presencia o ausencia de prótesis dentales, la adecuada higiene y si existe o no una enfermedad sistémica concomitante o condicionante.

OCAMPO, G. K. G. \& BASILIO, R. J. Oral microbiota in edentulous patients. Int. J. Odontostomat., 9(1):79-84, 2015.

ABSTRACT: The objective of this research was to identify oral conditions of edentulous patients attending rehabilitation at the Total Prosthetics Clinic, Faculty of Dentistry, University of the State of Mexico. The study design was observational, descriptive, transversal and prospective with a nonrandom sample and sequential opportunity to study in 160 patients the type of microbial flora. The results report that the average age for most edentulous population is between 56 and 65 years of age. Regarding existing in mouth pathologies found that $80 \%$ of the population did not present signs or symptoms, in the remaining $20 \%$ the symptoms most recognized is the pain with $50 \%$, ulcers in $30 \%$ and bleeding and inflammation by $10 \%$ respectively, all associated with denture stomatitis as the most frequently reported; burning mouth syndrome was reported in 2 of these patients' oral pathology. Regarding microbial flora of the oral cavity we found a variety of microorganisms which breaks down as follows: $17 \%$ for plants coccoid Gram +, $2 \%$ for plants bacillary Gram-, $17 \%$ of white blood cells, $18 \%$ of bacteria, $18 \%$ ofepithelial cells, $2 \%$ diplococci, $5 \%$ Staphylococcus, tetrads $7 \%, 7 \%$ of Micrococci, Streptococcus $5 \%, 2 \%$ Enterobactereceas. No yeast or Candida albicans were found. In conclusion, females are more prone to edentulism, national demographic reason. In the area of oral microflora a lot of abounding bacterial flora Gram + microorganisms identified, but several anaerobic organisms convert the oral cavity in a culture medium are identified, which must be correlated with age, presence of concomitant systemic disease, microorganismios as staphylococcus and streptococcus as can have an impact at the systemic level, as demonstrated by the association of edentulism cardiovascular and metabolic diseases.

KEY WORDS: edentulism, oral conditions, oral microbiota.

\section{REFERENCIAS BIBLIOGRÁFICAS}

Bagg, J. The oral micro flora and dental plaque. Essentials of microbiology for dental students. Oxford, Oxford University Press, 1999. pp.229-310.
Botero, J. E.; González, A. M.; Mercado, R. A.; Olave, G. \& Contreras, A. Subgingival microbiota in peri-implant mucosa lesions and adjacent teeth in partially edentulous 
patients. J. Periodontol., 76(9):1490-5, 2005.

Burnett, G. W.; Scherp, H. \& Schuster, G. Microbiología y enfermedades infecciosas de la boca. México D. F., Editorial Limusa, 1986. pp.277-319.

Danser, M. M.; Van Winkelhoff, A. J. \& van der Velden, U. Periodontal bacteria colonizing oral mucous membranes in edentulous patients wearing dental implants. $J$. Periodontol., 68(3):209-16, 1997.

Devides, S. L. \& Franco, A. T. Evaluation of peri-implant microbiota using the polymerase chain reaction in completely edentulous patients before and after placement of implant-supported prostheses submitted to immediate load. Int. J. Oral Maxillofac. Implants, 21(2):262-9, 2006.

Dinatale, E. \& Guilarte, C. Aspectos microbiológicos en implantología: Revisión de la literatura. Acta Odontol. Venez., 47(4):149-57, 2009.

Esponda Vila, R. Anatomía Dental. $6^{\mathrm{a}}$ ed. México D. F., Textos Universitarios UNAM, 1993. pp.19-29.

Koeck, B. Prótesis completas. $4^{\mathrm{a}}$ ed. Madrid, Elsevier Masson, 2007. pp.2-13.

Kostecka, F. Relation of the teeth to the normal development of the microbial flora in their oral cavity. Dental Cosmos, 66:927-35, 1924.

Kraus, B. S.; Abrams, L. \& Jordan, R. E. Anatomía dental y oclusión: un estudio del sistema masticatorio. México D. F., Nueva Editorial Interamericana, 1981. pp.189-201.

McCord, J. F. \& Grant, A. A. Pre-definitive treatment: rehabilitation prostheses. Br. Dent. J., 188(8):419-24, 2000.

Nolte, W. A. Microbiología odontológica: con nociones básicas de microbiología e inmunología. $3^{\mathrm{a}}$ ed. México D. F., Editorial Interamericana, 1982. pp.188-215.

Organización Panamericana de la Salud (OPS). La conexión entre la salud bucodental y las enfermedades cardiovasculares. Washington D. C., Organización Panamericana de la Salud (OPS), 2004. pp.109-13.

Ozawa Deguchi, J. Y. Prostodoncia Total. México D. F., Textos Universitarios UNAM, 1995. pp.39-46.

Regezi, J. \& Sciubba, J. Patología Bucal. Correlaciones Clinicopatológicas. México D. F., McGraw-Hill Interamericana Editores, 2004.
Dirección para Correspondencia:

Karla Gabriela Ocampo García

Catedrática de la Facultad de Odontología

Universidad Autónoma del Estado de México

Paseo Tollocan Esq. Jesús Carranza,

Col. Universidad

Toluca

Estado de México

C.P. 50000

MÉXICO

Email: pmfcoe@hotmail.com

Recibido : 04-08-2014

Aceptado: 04-03-2015 